\title{
Extensive squamous cell carcinoma of the lower lid
}

\author{
Boris Jančar \\ Department of Radiation Oncology, Institute of Oncology, Ljubljana, Slovenia
}

An 88-year-old patient was referred to our unit for the treatment of extensive squamous cell carcinoma of the lower lid of the right eye, extending to the lid and bulbar conjuctiva (Figures 1, 2).

Treatment proposed by ophthalmologist was resection of the lower lid with exenteration of the right eye. The patient was treated with the combination of teletherapy and brachitherapy. The lower lid and conjunctiva were treated by an ortovoltage unit with the dose of $70 \mathrm{~Gy}$, with the shielding of the globe. The bulbar conjunctiva was treated by an ophthalmic applicator with $90 \mathrm{Sr}$, with the dose of $60 \mathrm{~Gy}$ in four fractions. Complete regression of the tumour was achieved.

At the follow-up three years after the completed treatment, there was no local recurrence, distant metastases, or other evidence of disease. (Figures 3, 4).

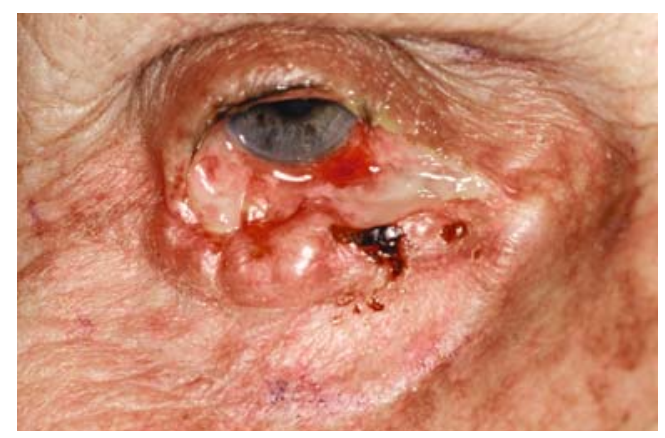

Figure 1. An extensive squamous cell carcinoma of the lower lid of the right eye.

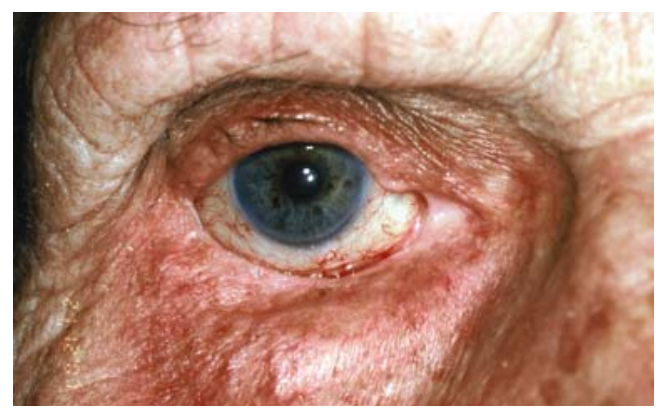

Figure 3. Three years after the completed treatment there was no evidence of disease.

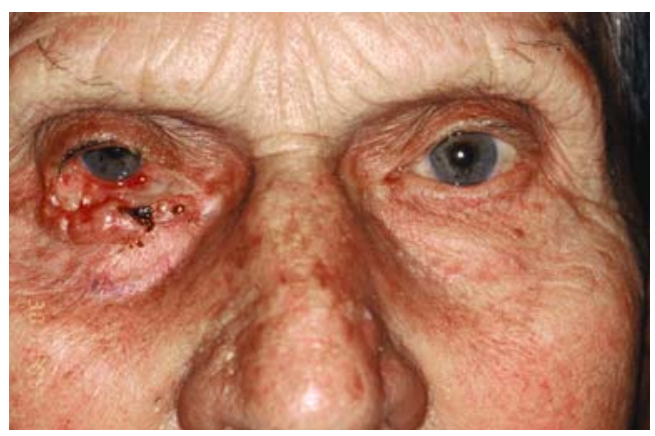

Figure 2. An extensive squamous cell carcinoma of the lower lid of the right eye.

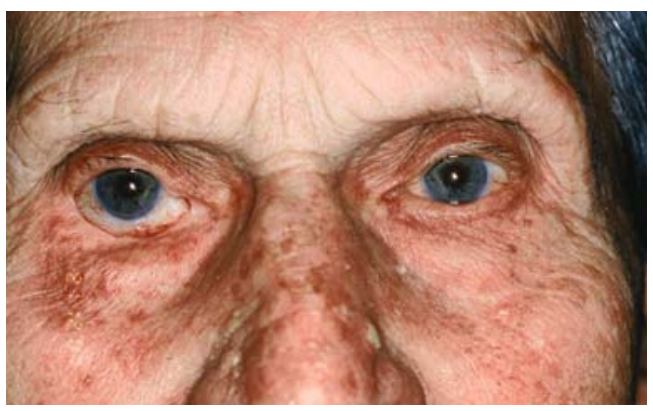

Figure 4. Three years after the completed treatment there was no local recurrence. 